\title{
Bile Acid Effects on Placental Damage in Intrahepatic Cholestasis of Pregnancy
}

\author{
Feng Xie*, Xiaofeng Liu*, Ping Xiao, Yang Huang, Qiaobing Chen, Li Zhou\# \\ Nanshan District Maternity \& Child Healthcare Hospital of Shenzhen, Shenzhen, China \\ Email: xiefeng101787@126.com, ${ }^{*}$ zhouli0712@126.com
}

How to cite this paper: Xie, F., Liu, X.F., Xiao, P., Huang, Y., Chen, Q.B. and Zhou, L. (2018) Bile Acid Effects on Placental Damage in Intrahepatic Cholestasis of Pregnancy. Journal of Biosciences and Medicines, 6, 42-52.

https://doi.org/10.4236/jbm.2018.66003

Received: March 21, 2018

Accepted: June 4, 2018

Published: June 7, 2018

Copyright $(0) 2018$ by authors and Scientific Research Publishing Inc. This work is licensed under the Creative Commons Attribution International License (CC BY 4.0).

http://creativecommons.org/licenses/by/4.0/

\begin{abstract}
Aims: The abnormal increase of bile acid is found in intrahepatic cholestasis of pregnancy (ICP). It also can be observed the damage of placental tissue in ICP. The aim of this study was to find the associations of the bile acid in umbilical vein and the damage of placental tissue. Methods: Thirty women diagnosed with ICP and fifty normal pregnant women between September 2015 and September 2017 at Nanshan District Maternity \& Child Healthcare Hospital of Shenzhen were included in this study. The glycocholic acid (GA), total bile acids (TBA), total bilirubin (TB), direct bilirubin (DB) and albumin level in umbilical vein were measured by cycle enzyme method in ICP and control group. The placental damage was analyzed by morphologic study using hematoxylin dyes in two groups. The correlation between the level of the bile acid in the umbilical vein and the damage of the placenta was assessed using SPSS software. Results: The GA, TBA, TB, DB and albumin level in umbilical vein were significantly higher in ICP than those of pregnant women, respectively. The placental villis were expanded and the structure was destroyed in ICP. The vessel was damaged and the cell trophoblast hyperplasia in ICP. It also can be seen that there was obvious nodules and a typical fibrous necrotic substance in ICP but not in control group. There is a positive correlation between the level of the TBA in the umbilical vein and the damage of the placenta in ICP. Conclusion: The TBAs were significantly higher in umbilical vein and were related to the placental damage in ICP.
\end{abstract}

\section{Keywords}

Intrahepatic Cholestasis of Pregnancy, Total Bile Acids, Placental Damage, Placental Villis, Trophoblast Hyperplasia

${ }^{\star}$ co-authors. 


\section{Introduction}

Intrahepatic cholestasis of pregnancy (ICP) is a disorder of pregnancy occurring in the third trimester, characterized by pruritus, accompanied by the elevation of serum transaminases and serum bile acids (SBA) [1]. The elevation of SBA has been associated with increased neonatal respiratory distress syndrome [2] [3], premature delivery [4], fetal asphyxia [5] and mortality [6]. The placenta has key roles in the regulation of fetal growth and development during pregnancy [7]. Although the pathogenesis and etiology of ICP have not been clarified, increasing evidence demonstrates that the placenta has a vital role in the development of ICP [8] [9], especially for the fetal BA metabolism and excretion [10].

During pregnancy, fetal liver synthesize primary bile acids (PBA), while the PBA can not be eliminated because of immaturity of fetal liver and kidney [11]. The placenta is the major route to eliminate fetal primary bile acids by transfering them to the maternal serum [12]. There is a transplacental gradient for bile acids in the fetal-to-mother direction [13]. And the bile acid in the fetus transported to the maternal serum through the placenta, actually need to cross the chorionic vascular endothelium, chorionic microvilli matrix and trophoblast [14]. Several experimental lines of evidence suggest that not only simple diffusion, but also the ATP-dependent transportation are the main mechanism by BA cross the human placenta, which depends on the bile acid transporter [15] [16].

In ICP, we have not only seen the abnormal increase of maternal serum bile acid, but also seen the damage of placenta, accompanied by the poor fetal outcome [17]. The fetal bile acid cannot transport to the maternal serum because of the damage of placenta and abnormal accumulate in the umbilical vein in ICP [18] [19]. Besides, the high level of primary bile acids is the biomarkers for the clinical of grade ICP [11] [20]. The relationship between the bile acids in umbilical vein and placental damage in intrahepatic cholestasis of pregnancy is still undefined, while the high level of bile acids in umbilical vein may be the reason for the damage of placenta.

In this pilot study, we raised a novel hypothesis that the high level of bile acids in umbilical vein damaged the placenta, even damaged the bile acid transporter in placenta, then lead the bile acid abnormal accumulate in the umbilical vein and cause to bad results. We compared the level of bile acid in umbilical vein between the ICP and control group. Subsequently, we also observed the damage of placental in ICP, especially the chorionic villis, the microvessels and trophoblast cells proliferation. Finally, we investigated the relationship between the level of bile acid in umbilical vein and the placental damage.

\section{Materials and Methods}

\subsection{Subjects}

Thirty consecutive pregnant women with ICP were enrolled in the study. A control group of 50 consecutive healthy women with physiological pregnancies were also studied as controls. The diagnosis of ICP was followed the guidelines for the 
management of intrahepatic cholestasis of pregnancy (2015) [21]. All pregnancies complicated by severe congenital malformations, consisting of chromosomal abnormalities and/or multiple congenital anomalies, and all twin pregnancies were excluded from the study. It also exclude some liver disease caused by alcohol, drug abuse, infectious, cholesterol gallstones, viral, or metabolic disorders.

\subsection{Placenta Morphology Detection}

Once the placenta is delivered, the central zone of the maternal face of placenta was collected and was saved in the $4^{\circ} \mathrm{C}$ physiological saline. Then the placenta was fixed with $4 \%$ formaldehyde, embedded by paraffin, and made into slices. The pathologic changes of placenta were observed by Optical microscope using hematoxylin dyes.

\subsection{Total Bile Acids in Umbilical Vein Detection}

At the time of delivery, the cord blood was collected and was centrifuged at 2000 $\mathrm{r} / \mathrm{min}$ for $15 \mathrm{~min}$, then saved in the $-20^{\circ} \mathrm{C}$. The GA, TBA, TB, DB and albumin were measured by enzymatic colorimetric determination.

\subsection{Correlation Analysis between the Total Bile Acids in Umbilical Vein and the Placenta Damage}

According to the placenta morphology of observation, the damage of placenta was classified by 3 grades. The correlation between the level of bile acid in the umbilical vein and the tissue injury of the placenta was assessed using Spearman's correlation coefficient.

\subsection{Statistical Analysis}

All data are presented as the mean \pm SD of the indicated number of measurements. The unpaired t test was used to determine the significance of two groups, with $\mathrm{P}<0.05$ as the cutoff for statistical significance. Correlations between TBA levels in umbilical vein and placental tissue damage were calculated using Spearman's correlation coefficient.

\section{Results}

\subsection{The Expression of Glycocholic Acid, Total Bile Acids (TBA), Total Bilirubin, Direct Bilirubin and Albumin Level in Umbilical Vein}

All the study populations were from September 2015 until September 2017 in Nanshan District Maternity \& Child Healthcare Hospital of Shenzhen. All the patients had an elective caesarean delivery. Baseline characteristics of the study population were shown in table 1 . Maternal age, and gestational age did not differ between women with ICP and women without ICP (Table 1).

When a pregnant woman delivers a cesarean section, once the placenta is delivered, we collected the cord blood and detected the level of GA, TBA, TB, DB 
Table 1. The maternal age, gestational age and neonatal sex of the two groups.

\begin{tabular}{ccccc}
\hline group & $\mathrm{n}$ & $\begin{array}{c}\text { Maternal Age } \\
(\mathrm{M} \pm \mathrm{SD} \text {, year })\end{array}$ & $\begin{array}{c}\text { Gestational Age } \\
(\mathrm{M} \pm \mathrm{SD} \text {, week })\end{array}$ & $\begin{array}{c}\text { Gender } \\
\text { (male/female) }\end{array}$ \\
\hline control & 50 & $32.24 \pm 3.58$ & $39.28 \pm 1.26$ & $25 / 25$ \\
ICP & 30 & $30.77 \pm 5.15$ & $38.34 \pm 1.55$ & $18 / 12$ \\
\hline
\end{tabular}

(Data are mean $\pm \mathrm{SD}$ of 4 independent experiments).

and albumin in umbilical cord. We found that TBA, GA, TB, DB and albumin level in ICP were significantly higher than those of pregnant women (Table 2).

\subsection{The Damage of Placental in ICP Patients}

Once the placenta is delivered, the pathological tissue section was made by the fresh tissue of $2 \mathrm{~cm} \times 2 \mathrm{~cm} \times 2 \mathrm{~cm}$ in the central zone of the maternal face of the placenta in the sterile condition. The pathologic changes of placenta were observed by Optical microscope using hematoxylin dyes. In microscope, some of the complete chorionic villis, with small volume, and little exudations were observed in the control group (Figure 1(A)); while in ICP group, the structure of the chorionic villis was destroyed and dilated, the exudations are increased (Figure 1(B)).

The control group can be seen with intact microvessels and red blood cells in the vessels (Figure 2(A)); while in ICP group, the microvessels are damaged in placenta, and there is no complete vascular structure, only see the sinus (Figure 2(B)). All these showed that microvascular damage is observed in ICP patients placenta.

In control group, the complex nodules are not obvious (Figure 3(A)), the cell trophoblast cells are normal (Figure 3(C)), and the fibroid necrosis is rarely seen (Figure 3(E)); while in ICP group, the complex nodules are more obvious (Figure 3(B)), and the trophoblast cells proliferate (Figure 3(D)), and there is a typical fibrinoid necrotic material deposition (Figure $3(\mathrm{~F})$ ).

\subsection{Correlation Analysis between Total Bile Acid Level and Placental Tissue Damage in ICP}

The damage of the placenta of 30 ICP patients was observed under an optical microscope, and the results were followed in the Table 3. The correction between total bile acid level and placental tissue damage was assessed by SPSS. The results showed that the total bile acid was positively correlated with the damage of placenta, and the correlation coefficient was 0.889 (Figure 4).

\section{Discussion}

It was proved that fetal physiological cholestasis exists and the concentration of serum bile acid in maternal and fetal circulation was significantly increased in ICP [22] [23]. The concentration of serum bile acid in maternal acts as an indicator for the diagnosis and determination of ICP, while the fetal bile acid could 
Table 2. The expression of glycocholic acid, total bile acids (TBA), total bilirubin, direct bilirubin and albumin level in two groups.

\begin{tabular}{ccccccc}
\hline group & $\mathbf{n}$ & GA & TBA & TB & DB & albumin \\
\hline control & 50 & $0.53 \pm 0.63$ & $4.26 \pm 1.89$ & $8.03 \pm 3.27$ & $2.09 \pm 1.92$ & $5.94 \pm 1.76$ \\
& & $10.07 \pm$ & $26.10 \pm$ & $21.39 \pm$ & $6.70 \pm$ & $14.69 \pm$ \\
ICP & \multirow{2}{*}{30} & $11.62^{* *}$ & $18.84^{* *}$ & $12.35^{* *}$ & $5.15^{* * *}$ & $8.77^{* * *}$ \\
\hline
\end{tabular}

(Data are mean $\pm \mathrm{SD}$ of 4 independent experiments. ${ }^{*} \mathrm{P}<0.05$ vs control group; ${ }^{* *} \mathrm{P}<0.0001$ vs control group).

control group

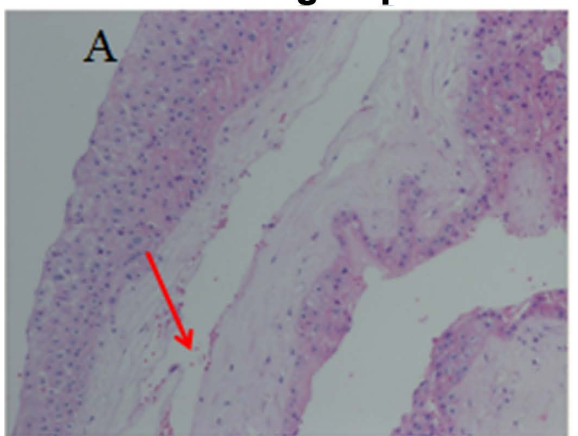

ICP group

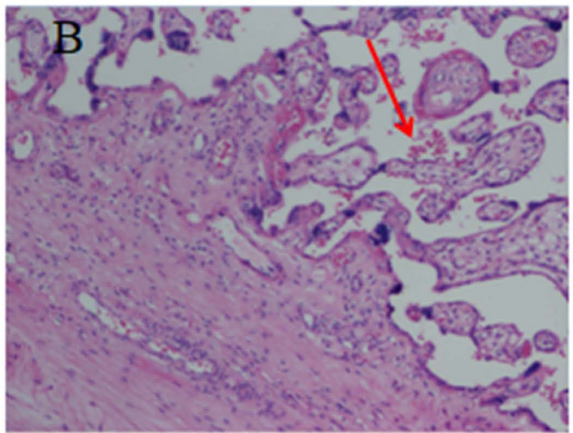

Figure 1. The damage of the placental villi (hematoxylin dyes $10 \times 10$; red arrow: exudations.

control group

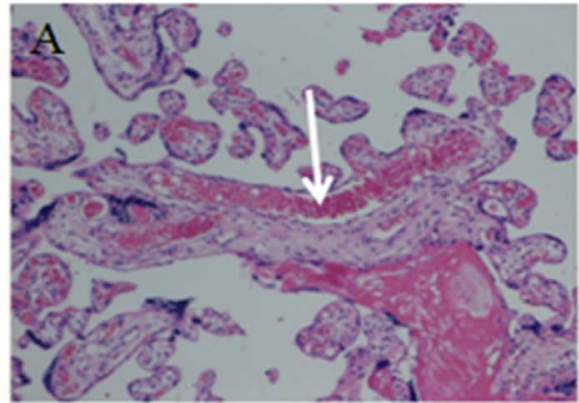

ICP group

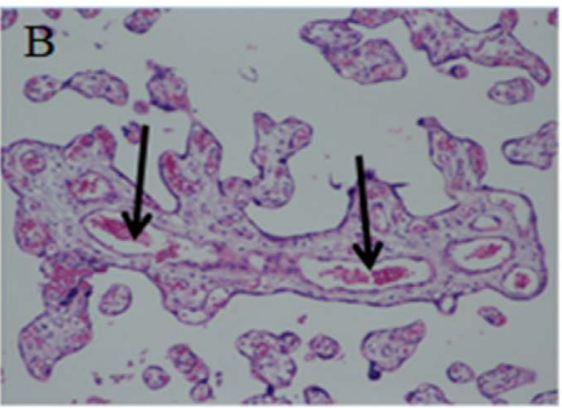

Figure 2. The microvessels in the placental (hematoxylin dyes $10 \times 10$; white arrow: microvessels; black arrow: sinus).

not be reflected because of pregnancy [13]. In our results, we detected the bile acid in umbilical cord to find the level of fetal bile acid when the time of the placenta is delivered. We found that the levels of TBA in umbilical cord abnormal increased in ICP than control group. Not only TBA, but also the expression of GA, TB, DB and albumin level all increased in umbilical cord. It is reported that a high rate of fetal complications was related to a TBA in maternal serum level of more than $40 \mu \mathrm{mol} / \mathrm{L}$ [13]. In our results, the TBA in umbilical cord is $26.10 \pm$ $18.84 \mu \mathrm{mol} / \mathrm{L}$, really lower to $40 \mu \mathrm{mol} / \mathrm{L}$. But it is not clearly that the relationship of TBA between in maternal serum and in umbilical cord. Further research is needed to confirm that if the high level of bile acid in umbilical cord is also related to fetal complications. 

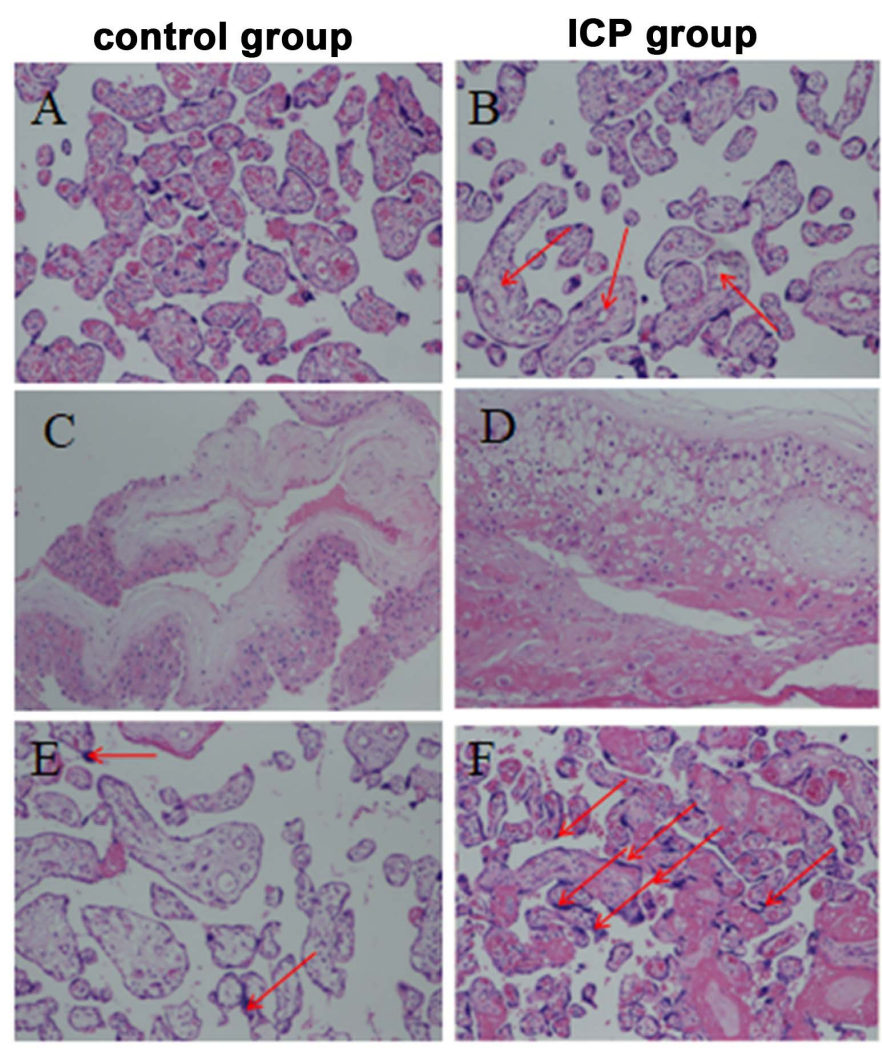

Figure 3. The damages in the placental (hematoxylin dyes $10 \times 10$ ).

In adults, bile acid metabolism and excretion are mainly through the liver and kidneys [24]. During pregnancy, the placenta as a bile acid barrier and the excretion of bile acids mainly depends on the placenta [25] [26]. There are several papers showed that placentas from women with ICP are associated with morphological abnormalities, such as increased occurrence of syncytial knots, thought to represent areas of apoptosis, reduced intervillous space and smaller blood vessels [27] [28] [29]. In our results, we also observed the morphological changes in placenta of patients with ICP. The chorionic villis were destroyed and the whole chorionic vessels could not be seen in ICP patient placenta. We also observed that the complex nodules are generated, the trophoblast stromal cells abnormal proliferated and the typical fibrinoid necrotic material deposited in ICP. These structural changes in the placenta, implicating bile acids in the pathology, can have severe consequences for the fetus.

There are many reports showed that the rise in bile acids in the feto-placental unit that occurs in ICP has multiple detrimental effects on the placenta [30] [31] [32]. The influence of high bile acid on placental tissues is mainly manifested in: The toxicity of high bile acid level can direct damage the placental tissue, induce the trophoblast cells apoptosis, also damage the placental chorionic veins, affecting the fetal and placental blood circulation [33] [34] [35]. Futhermore, some reports showed that the high bile acid levels damaged the placenta by induced hypoxia or inflammation [36] [37]. We used SPSS to detect the correlation 
Table 3. The total bile acids and the damage of the placenta of 30 ICP patients.

\begin{tabular}{|c|c|c|c|c|}
\hline number & Maternal age & Gestational age & TBA & Placental damage leve \\
\hline 1 & Y27 & $38 w+5$ & 42 & 3 \\
\hline 2 & Y24 & $38 w+2$ & 38 & 2 \\
\hline 3 & Y24 & $37 w+5$ & 74 & 3 \\
\hline 4 & Y34 & $38 \mathrm{w}$ & 70 & 3 \\
\hline 5 & Y29 & $40 w$ & 42 & 2 \\
\hline 6 & Y30 & $41 w+1$ & 18 & 1 \\
\hline 7 & Y35 & $35 w+3$ & 36 & 2 \\
\hline 8 & Y30 & $39 w+5$ & 33 & 2 \\
\hline 9 & Y23 & $35 w+4$ & 14 & 1 \\
\hline 10 & Y34 & $36 w+5$ & 27 & 1 \\
\hline 11 & Y43 & $34 w+4$ & 25 & 2 \\
\hline 12 & Y29 & $37 w+6$ & 16 & 1 \\
\hline 13 & Y27 & $39 w+2$ & 21 & 2 \\
\hline 14 & Y37 & $40 w+2$ & 20 & 2 \\
\hline 15 & Y29 & $37 w+1$ & 15 & 1 \\
\hline 16 & Y34 & $38 w+3$ & 61 & 3 \\
\hline 17 & Y23 & $38 \mathrm{w}$ & 17 & 1 \\
\hline 18 & Y30 & $39 w$ & 10 & 1 \\
\hline 19 & Y33 & $37 w+4$ & 10 & 1 \\
\hline 20 & Y34 & $38 w+2$ & 7 & 1 \\
\hline 21 & Y27 & $40 w+3$ & 6 & 1 \\
\hline 22 & Y34 & $39 w+4$ & 19 & 1 \\
\hline 23 & Y39 & $40 w+2$ & 8 & 1 \\
\hline 24 & Y36 & $39 w+1$ & 10 & 1 \\
\hline 25 & Y36 & $39 w+1$ & 30 & 2 \\
\hline 26 & Y21 & $38 w+6$ & 16 & 2 \\
\hline 27 & Y29 & $38 w+2$ & 4 & 1 \\
\hline 28 & Y34 & $36 w$ & 9 & 1 \\
\hline 29 & Y28 & $39 w$ & 50 & 3 \\
\hline 30 & Y30 & $40 w+1$ & 35 & 2 \\
\hline
\end{tabular}

between total bile acid level and placental tissue damage and found there is a positively correlation between the total bile acid level and placental tissue damage. Several experiments have provided evidence that a causal relationship between levels of BA and adverse fetal outcome [10] [38]. The accumulation of bile acid in the placenta directly damage the placenta tissue, influence the material exchange between fetus and mother, lead to the supply of nutrients and oxygen barrier fetus, and jeopardize fetal health. 


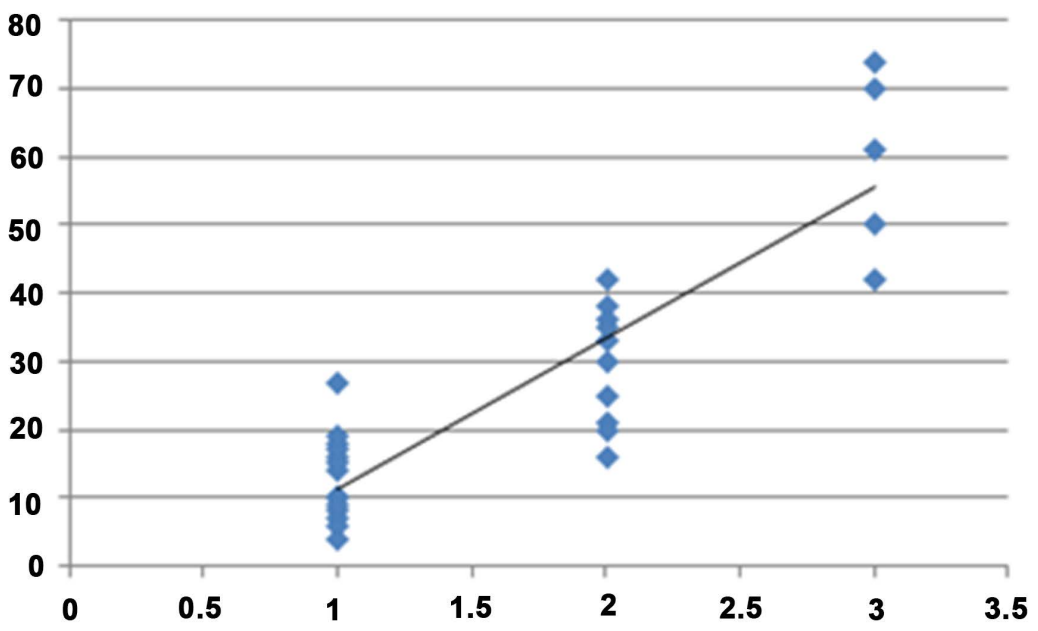

Figure 4. The correlations between total bile acids and the damage of the placenta $\left(\mathrm{r}^{2}=0.889, \mathrm{p}<0.001\right)$.

We need to highlight some limitations of this study. First of all, all the study populations we selected with cesarean section and did not include patients with vaginal delivery. Then, we do not demonstrate the relationship of TBA between in maternal serum and in umbilical cord. Moreover, we do not found what is the normal value of TBA in umbilical cord and what is the risk value of TBA in umbilical cord to cause placental damage. In addition, we do not test the molecular mechanism of high bile acid causes placental damage.

\section{Conclusion}

The level of total bile acid in umbilical vein was significantly higher in ICP than those of pregnant women. And we add an important new piece of information revealing that there is a positive correlation between the level of the bile acid in the umbilical vein and the damage of placenta in ICP which includes the direct damage of chorionic villis/the destroy of blood vessels/the abnormal proliferation of trophoblast stromal cells. Our results suggest that the high levels of total bile acid in umbilical cord damaged the placenta, and broke the bile acid circulation between the fetal and maternal, ended up dangers of maternal and fetus.

\section{Fund}

This work was supported by grants from the Shenzhen Nanshan district science and technology project. Project was funded by Nanshan district health and family planning bureau (2015007).

\section{Author Contribution}

Conceived and designed the experiments: Feng Xie, Li Zhou, Xiaofeng Liu. Performed the experiments: Feng Xie, Qiaobing Chen, Yang Huang. Analyzed the data: Feng Xie. Modified the language: Li Zhou. 


\section{References}

[1] Floreani, A. and Gervasi, M.T. (2016) New Insights on Intrahepatic Cholestasis of Pregnancy. Insights on Intrahepatic Cholestasis of Pregnancy, 20, 177-189. https://doi.org/10.1016/j.cld.2015.08.010

[2] Zecca, E., De Luca, D., Marras, M., et al. (2006) Intrahepatic Cholestasis of Pregnancy and Neonatal Respiratory Distress Syndrome. Pediatrics, 117, 1669-1672. https://doi.org/10.1542/peds.2005-1801

[3] Zecca, E., De Luca, D., Baroni, S., et al. (2008) Bile Acid-Induced Lung Injury in Newborn Infants: A Bronchoalveolar Lavage Fluid Study. Pediatrics, 121, 141-149. https://doi.org/10.1542/peds.2007-1220

[4] Kondrackiene, J., Beuers, U., Zalinkevicius, R., et al. (2014) Predictors of Premature Delivery in Patients with Intrahepatic Cholestasis of Pregnancy. BMC Medical Genomics, 7, 42-53.

[5] Wikström Shemer, E.A., Thorsell, M., Marschall, H.U., et al. (2013) Risks of Emergency Cesarean Section and Fetal Asphyxia after Induction of Labor in Intrahepatic Cholestasis of Pregnancy: A Hospital-Based Retrospective Cohort Study. Sexual \& Reproductive Healthcare, 4, 17-22. https://doi.org/10.1016/j.srhc.2012.11.005

[6] Zecca, E., De Luca, D., Barbato, G., et al. (2008) Predicting Respiratory Distress Syndrome in Neonates from Mothers with Intrahepatic Cholestasis of Pregnancy. Early Human Development, 84, 337-341. https://doi.org/10.1016/j.earlhumdev.2007.09.012

[7] Salafia, C.M., Charles, A.K. and Maas, E.M. (2006) Placenta and Fetal Growth Restriction. Clinical Obstetrics \& Gynecology, 49, 236-256. https://doi.org/10.1097/00003081-200606000-00007

[8] Du, Q., Pan, Y., Zhang, Y., et al. (2014) Placental Gene-Expression Profiles of Intrahepatic Cholestasis of Pregnancy Reveal Involvement of Multiple Molecular Pathways in Blood Vessel Formation and Inflammation. BMC Medical Genomics, 7, 42-53. https://doi.org/10.1186/1755-8794-7-42

[9] He, P., Wang, F., Jiang, Y., et al. (2014) Placental Proteome Alterations in Women with Intrahepatic Cholestasis of Pregnancy. International Journal of Gynecology and Obstetrics, 126, 256-259. https://doi.org/10.1016/j.ijgo.2014.03.035

[10] Marin, J.J., Serrano, M.A., El-Mir, M.Y., et al. (1990) Bile Acid Transport by Basal Membrane Vesicles of Human Term Placental Trophoblast. Gastroenterology, 99, 1431-1438. https://doi.org/10.1016/0016-5085(90)91172-3

[11] Chen, J., Deng, W., Wang, J., et al. (2013) Primary Bile Acids as Potential Biomarkers for the Clinical Grading of Intrahepatic Cholestasis of Pregnancy. International Journal of Gynecology and Obstetrics, 122, 5-8. https://doi.org/10.1016/j.ijgo.2013.02.015

[12] Marin, J.J., Macias, R.I., Briz, O., et al. (2008) Molecular Bases of the Fetal Liverplacenta-Maternal Liver Excretory Pathway for Cholephilic Compound. Liver International, 28, 435-454. https://doi.org/10.1111/j.1478-3231.2008.01680.x

[13] Geenes, V., Lövgren-Sandblom, A., Benthin, L., et al. (2014) The Reversed Feto-Maternal Bile Acid Gradient in Intrahepatic Cholestasis of Pregnancy Is Corrected by Ursodeoxycholic Acid. PLoS ONE, 9, e83828. https://doi.org/10.1371/journal.pone.0083828

[14] Macias, R.I., Marin, J.J. and Serrano, M.A. (2009) Excretion of Biliary Compounds during Intrauterine Life. World Journal of Gastroenterology, 15, 817-828.

https://doi.org/10.3748/wjg.15.817 
[15] Bravo, P., Marin, J.J., Beveridge, M.J., et al. (1995) Reconstitution and Characterization of ATP-Dependent Bile acid Transport in Human and Rat Placenta. Biochemistry, 311, 479-485. https://doi.org/10.1042/bj3110479

[16] Hagenbuch, B. and Meier, P.J. (2004) Organic Anion Transporting Polypeptides of the OATP/SLC21 Family: Phylogenetic Classification as OATP/SLCO Superfamily, New Nomenclature and Molecular/Functional Properties. Pflügers Archiv, 447, 653-665.

[17] Kondrackiene, J. and Kupcinskas, L. (2008) Intrahepatic Cholestasis of Pregnancy-Current Achievements and Unsolved Problems. World Journal of Gastroenterology, 14, 5781-5788. https://doi.org/10.3748/wjg.14.5781

[18] Marin, J.J., Macias, R.I. and Serrano, M.A. (2003) The Hepatobiliary-Like Excretory Function of the Placenta. Placenta, 24, 431-438. https://doi.org/10.1053/plac.2002.0951

[19] Serrano, M.A., Macias, R.I., Briz, O., et al. (2007) Expression in Human Trophoblast and Choriocarcinoma Cell Lines, BeWo, Jeg-3 and Jar of Genes Involved in the Hepatobiliary-Like Excretory Function of the Placenta. Placenta, 28, 107-117.

[20] Favre, N., Bourdel, N., Sapin, V., et al. (2010) Importance of Bile Acids for Intra-Hepatic Cholestasis of Pregnancy. Gynecologie, Obstetrique \& Fertilite, 38, 293-295. https://doi.org/10.1016/j.gyobfe.2010.02.011

[21] Obstetrics Subgroup, Chinese Society of Obstetrics and Gynecology, Chinese Medical Association (2015) Guidelines for the Management of Intrahepatic Cholestasis of Pregnancy. Chinese Journal of Obstetrics and Gynecology, 50, 481-485.

[22] Rook, M., Vargas, J., Caughey, A., et al. (2012) Fetal Outcomes in Pregnancies Complicated by Intrahepatic Cholestasis of Pregnancy in a Northern California Cohort. PLoS ONE, 7, e28343.

[23] Monte, M.J., Rodriguez-Bravo, T., Macias, R.I., et al. (1995) Relationship between Bile Acid Transplacental Gradients and Transport across the Fetal-Facing Plasma Membrane of the Human Trophoblast. Pediatric Research, 38, 156-163. https://doi.org/10.1203/00006450-199508000-00004

[24] Glantz, A., Marschall, H. and Mattsson, L. (2010) Intrahepatic Cholestasis of Pregnancy: Relationships between Bile Acid Levels and Fetal Complication Rates. Hepatology, 40, 467-474. https://doi.org/10.1002/hep.20336

[25] Maillette de Buy Wenniger, L. and Beuers, U. (2010) Bile Salts and Cholestasis. Digestive and Liver Disease, 42, 409-418. https://doi.org/10.1016/j.dld.2010.03.015

[26] Myllynen, P., Immonen, E., Kummu, M., et al. (2009) Developmental Expression of Drug Metabolizing Enzymes and Transporter Proteins in Human Placenta and Fetal Tissues. Expert Opinion on Drug Metabolism \& Toxicology, 5, 1483-1499. https://doi.org/10.1517/17425250903304049

[27] Geenes, V.L., Lim, Y.-H., Bowman, N., et al. (2011) A Placental Phenotype for Intrahepatic Cholestasis of Pregnancy. Placenta, 32, 1026-1032.

[28] Mcllvride, S. and Dixon, P.H. (2017) Catherine Williamson. Bile Acids and Gestation. Molecular Aspects of Medicine, 56, 90-100.

[29] Wikström Shemer, E., Thorsell, M., Östlund, E., et al. (2012) Stereological Assessment of Placental Morphology in Intrahepatic Cholestasis of Pregnancy. Placenta 33, 914-918.

[30] Du, Q.L., Pan, Y., Zhang, Y.H., et al. (2014) Placental Gene-Expression Profiles of Intrahepatic Cholestasis of Pregnancy Reveal Involvement of Multiple Molecular Pathways in Blood Vessel Formation and Inflammation. BMC Medical Genomics, 
7, 42-53.

[31] He, P., Wang, F., Jiang, Y., et al. (2014) Placental Proteome Alterations in Women with Intrahepatic Cholestasis of Pregnancy. International Journal of Gynecology and Obstetrics, 126, 256.

[32] Wei, J., Wang, H., Yang, X., et al. (2010) Altered Gene Profile of Placenta from Women with Intrahepatic Cholestasis of Pregnancy. Archives of Gynecology and Obstetrics, 281, 801-810. https://doi.org/10.1007/s00404-009-1156-3

[33] Beninati, M., Seferovic, M.D., Liu, X., et al. (2016) The Placenta and Intrahepatic Cholestasis of Pregnancy (IHCP, Alterations to Bile Acid Transport Associated with Disease Duration and Reduction in Primary Bile Acid Concentrations. American Journal of Obstetrics and Gynecology, 214, S272-S272.

[34] Sepúlveda, W.H., González, C., Cruz, M.A., et al. (1991) Vasoconstrictive Effect of Bile Acids on Isolated Human Placental Chorionic Veins. European Journal of $O b-$ stetrics \& Gynecology and Reproductive Biology, 42, 211-215. https://doi.org/10.1016/0028-2243(91)90222-7

[35] Abu-Hayyeh, S., Papacleovoulou, G. and Williamson, C. (2013) Nuclear Receptors, Bile Acids and Cholesterol Homeostasis Series-Bile Acids and Pregnancy. Molecular and Cellular Endocrinology, 368, 120-128.

[36] Zhang, Y., Pan, Y., Lin, C., et al. (2016) Bile Acids Evoke Placental Inflammation by Activating Gpbar1/NF- $\kappa \mathrm{B}$ Pathway in Intrahepatic Cholestasis of Pregnancy. Journal of Molecular Cell Biology, 8, 530-541.

[37] Mella, M.T., Kohari, K., Jones, R., et al. (2016) Mitochondrial Gene Expression Profiles Are Associated with Intrahepatic Cholestasis of Pregnancy. Placenta, 45, 16-23.

[38] Rezai, S., Lam, J. and Henderson, C.E. (2015) Intrahepatic Cholestasis of Pregnancy: Maternal and Fetal Outcomes Associated with Elevated Bile Acid Levels. American Journal of Obstetrics and Gynecology, 213, 114.

https://doi.org/10.1016/j.ajog.2015.03.040 